\title{
A STRAIN ENERGY DERIVATION OF THE TORSIONAL- FLEXURAL BUCKLING LOADS OF STRAIGHT COLUMNS OF THIN-WALLED OPEN SECTIONS*
}

\author{
BY \\ N. J. HOFF \\ Polytechnic Institute of Brooklyn
}

In the thin-walled open section columns of modern aluminum alloy aircraft torsional buckling and combinations of torsional and flexural buckling are of consider. able importance. The critical loads corresponding to these types of instability have been calculated by Wagner, ${ }^{1}$ Kappus, ${ }^{2}$ Lundquist and Fligg, ${ }^{3}$ and Goodier ${ }^{4}$ through integrating the differential equations of the problem. In the present paper the torsional-flexural buckling loads are determined with the aid of the Rayleigh-RitzTimoshenko method. This procedure obviates the derivation and integration of the differential equations as well as the geometric considerations connected with what Goodier termed "Wagner's hypothesis."

The equilibrium of a straight bar of a length $L$ and a cross-sectional area $A$, loaded axially with a compressive force of a magnitude $\sigma A$ distributed uniformly over the end section, can be investigated by assuming that each section of the bar undergoes a virtual displacement. The end sections of the bar are assumed to be restrained in a manner which precludes translations as well as rotations about any axis perpendicular to the end section, but which permits rotations about axes in the plane of the end section and warping of the end section. Barring displacements that would change the shape of the cross section (such displacements lead to plate- or shell-buckling), the most general virtual displacement pattern of the bar can be represented by the following infinite series:

$$
\begin{aligned}
u & =\sum_{n=1}^{\infty} a_{n} \sin (n \pi z / L), \\
v & =\sum_{n=1}^{\infty} b_{n} \sin (n \pi z / L), \\
\beta & =\sum_{n=1}^{\infty} c_{n} \sin (n \pi z / L) .
\end{aligned}
$$

* Received July 24, 1943.

1 Wagner, Herbert, Verdrehung und Knickung von offenen Profilen, 25th Anniversary Volume of the Technische Hochschule, Danzig, 1929, p. 329.

Torsion and buckling of open sections, N.A.C.A. Tech. Mem., No. 807, 1936.

2 Kappus, Robert, Drillknicken zentrisch gedrückter Stäbe mit offenem Profil im elastischen Bereich, Luftfahrtforschung, 14, 44 (1937). Mem., No. 851, 1938.

Twisting failure of centrally loaded open-section columns in the elastic range, N.A.C.A. Tech.

${ }^{3}$ Lundquist, E. E., and Fligg, C. M., A theory for primary failure of straight centrally loaded columns, N.A.C.A. Report No. 582, 1937.

- Goodier, J. N., The buckling of compressed bars by torsion and flexure, Cornell University Engineering Experiment Station, Bulletin No. 27, December, 1941.

Torsional and flexural buckling of bars of thin-walled open section under compressive and bending loads, Transactions A.S.M.E., 64, A-103 (1942). 
In these equations $u$ is the virtual translation in the $x$-direction of a section of the bar at a distance $z$ from the bottom section, $v$ that in the $y$-direction, and $\beta$ the virtual rotation of the section about its shear center. The $x$ - and $y$-axes are parallel to the principal axes of inertia, and pass through the shear center of each section. The displacements of a section are shown in Fig. 1.

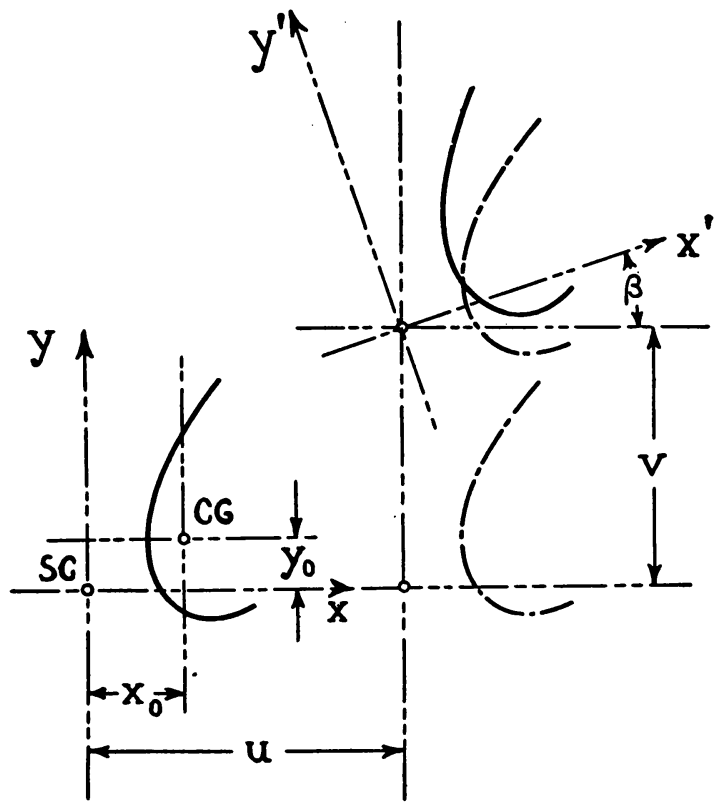

Fig. 1.

The increment $\delta U$ of the strain energy because of the virtual displacements is

$$
\begin{aligned}
\delta U= & \frac{1}{2} E I_{y} \int_{0}^{L}\left(d^{2} u / d z^{2}\right)^{2} d z+\frac{1}{2} E I_{x} \int_{0}^{L}\left(d^{2} v / d z^{2}\right)^{2} d z \\
& +\frac{1}{2} G C \int_{0}^{L}(d \beta / d z)^{2} d z+\frac{1}{2} E \Gamma \int_{0}^{L}\left(d^{2} \beta / d z^{2}\right)^{2} d z .
\end{aligned}
$$

In Eq. (2) $E I_{x}$ and $E I_{y}$ stand for the bending rigidities of the bar when the bending moment vector is parallel to the $x$-axis and the $y$-axis, respectively, and $G C$ is the torsional rigidity calculated from the Saint-Venant theory of uniform torsion. The fourth term is due to the direct stress caused by non-uniform warping, and $\Gamma$ is the warping constant defined in the theory of non-uniform torsion of thin-walled open sections. This theory is discussed in the previously mentioned references and in a paper written by the author. ${ }^{5}$ Eq. (2) follows from the theory of non-uniform torsion of thin-walled open sections and from the Bernoulli-Euler theory of bending, if the strain energy due to shear associated with bending and that associated with nonuniform warping are neglected.

'Hoff, N. J., Stresses in space-curved rings reinforcing the edges of cut-outs in monocoque fuselages, Journal Roy. Aeron. Soc., 47, 64 (1943). 
Because of the orthogonal properties of the trigonometric functions the integrals indicated in Eq. (2) can be easily calculated:

$$
\begin{aligned}
\delta U= & \left(\pi^{4} E I_{y} / 4 L^{3}\right) \sum_{n=1}^{\infty} n^{4} a_{n}^{2}+\left(\pi^{4} E I_{x} / 4 L^{3}\right) \sum_{n=1}^{\infty} n^{4} b_{n}^{2} \\
& +\left(\pi^{4} E \Gamma / 4 L^{3}\right) \sum_{n=1}^{\infty} n^{4} c_{n}^{2}+\left(\pi^{2} G C / 4 L\right) \sum_{n=1}^{\infty} n^{4} c_{n}^{2} .
\end{aligned}
$$

The decrease $-\delta V$ of the potential of the external forces is equal to the work $\delta W$ done by them during the virtual displacements. The work $d \delta W$ done by the infinitesimal force $\sigma d A$ is equal to the force times the shortening of the distance between the end points of the fiber upon which it is acting. The shortening $\Delta L_{x, y}$ of the distance between the end points of the fiber passing through a point $x, y$ can be calculated from the equation

$$
\Delta L_{x, y}=\frac{1}{2} \int_{0}^{L}\left[\left(d u_{x, y} / d z\right)^{2}+\left(d v_{x, y} / d z\right)^{2}\right] d z .
$$

It may be seen from Fig. 1 that for small displacements

$$
\begin{aligned}
& u_{x, y}=u-y \beta, \\
& v_{x, y}=v+x \beta .
\end{aligned}
$$

Upon substitution of the expressions of equations (1) and (5) into Eq. (4), integration yields

$$
\begin{aligned}
\Delta L_{x, y}= & \left(\pi^{2} / 4 L\right)\left\{\sum_{n=1}^{\infty} n^{2} a_{n}^{2}+\sum_{n=1}^{\infty} n^{2} b_{n}^{2}-2 y \sum_{n=1}^{\infty} n^{2} a_{n} c_{n}\right. \\
& \left.+2 x \sum_{n=1}^{\infty} n^{2} b_{n} c_{n}+x^{2} \sum_{n=1}^{\infty} n^{2} c_{n}^{2}+y^{2} \sum_{n=1}^{\infty} n^{2} c_{n}^{2}\right\} .
\end{aligned}
$$

The sum $\delta W$ of the work done by all the infinitesimal forces $\sigma d A$ is

$$
\delta W=\int_{A} \sigma \Delta L_{x, y} d A,
$$

where the integral is extended over the total cross-sectional area. With

$\int_{A} \sigma d A=P$, the total compressive force,

$\int_{A} y d A=y_{0} A$, the static moment of the section with respect to the $x$-axis passing through the shear center,

$\int_{A} x d A=x_{0} A$, the static moment of the section with respect to the $y$-axis passing through the shear center,

$\int_{A}\left(x^{2}+y^{2}\right) d A=\int_{A} r^{2} d A=I_{p}$, the polar moment of inertia of the section with respect to the shear center, and

equation (7) can be written as

$$
\rho^{2}=I_{p} / A
$$

$$
\begin{aligned}
\delta W= & \left(\pi^{2} P / 4 L\right)\left\{\sum_{n=1}^{\infty} n^{2} a_{n}^{2}+\sum_{n=1}^{\infty} n^{2} b_{n}^{2}-2 y_{0} \sum_{n=1}^{\infty} n^{2} a_{n} c_{n}\right. \\
& \left.+2 x_{0} \sum_{n=1}^{\infty} n^{2} b_{n} c_{n}+\rho^{2} \sum_{n=1}^{\infty} n^{2} c^{2}\right\} .
\end{aligned}
$$


According to the principle of virtual displacements the bar is in equilibrium in its original straight-line form if the change of the total potential $\delta(U+V)=\delta U-\delta W$ is zero for any virtual displacement provided that first order small terms alone are considered. Since both $\delta U$ (Eq. (3)) and $\delta W$ (Eq. (8)) contain only second order terms in the $a_{n}, b_{n}, c_{n}$, the original straight-line form is a configuration of equilibrium. This equilibrium is stable only if the total potential increases, that is $\delta(U+V)$ is positive, for any virtual displacement. With the notation

$$
\begin{aligned}
N & =\pi^{2} E I_{y} / L^{2}, \\
Q & =\pi^{2} E I_{x} / L^{2}, \\
R & =\pi^{2} E \Gamma / L^{2},
\end{aligned}
$$

the increment of the total potential can be written in the form

$$
\begin{aligned}
\delta(U+V)= & \left(\pi^{2} P / 4 L\right) \sum_{n=1}^{\infty} n^{2}\left\{\left[n^{2}(N / P)-1\right] a_{n}^{2}+\left[n^{2}(Q / P)-1\right] b_{n}^{2}\right. \\
& \left.+\left[n^{2}(R / P)+(G C / P)-\rho^{2}\right] c_{n}^{2}+2 y_{0} a_{n} c_{n}-2 x_{0} b_{n} c_{n}\right\} .
\end{aligned}
$$

With the notation

$$
\begin{aligned}
& A_{n}=n^{2}(N / P)-1, \quad B_{n}=n^{2}(Q / P)-1, \\
& C_{n}=n^{2}(R / P)+(G C / P)-\rho^{2}, \\
& F_{n}=-x_{0}, \quad G_{n}=y_{0}, \\
& X_{n}=A_{n} a_{n}^{2}+B_{n} b_{n}^{2}+C_{n} c_{n}^{2}+2 F_{n} b_{n} c_{n}+2 G_{n} c_{n} a_{n}
\end{aligned}
$$

the infinite sum on the right hand side can be written as $\sum_{n=1}^{\infty} X_{n}$. The necessary and sufficient conditions for its positive definite character are that all $X_{n}$ for $n=1,2, \ldots$ must be positive definite. Necessary and sufficient conditions for this are

or,

$$
A_{n}>0, \quad B_{n}>0, \quad A_{n} B_{n} C_{n}-A_{n} F_{n}^{2}-B G_{n}^{2}>0
$$

$$
\begin{aligned}
& \rho^{2}\left[n^{2}(N / P)-1\right]\left[n^{2}(Q / P)-1\right][(T / P)-1] \\
& \quad-x_{0}^{2}\left[n^{2}(N / P)-1\right]-y_{0}^{2}\left[n^{2}(Q / P)-1\right]>0 .
\end{aligned}
$$

Since inequalities (14)-(16) are necessary as well as sufficient conditions of stability, the bar may buckle if any one of them is not satisfied. Neutral equilibrium prevails, therefore, if any one of the following "buckling conditions" is fulfilled:

$$
\begin{gathered}
n^{2}(N / P)-1=0, \\
n^{2}(Q / P)-1=0, \\
\rho^{2}\left[n^{2}(N / P)-1\right]\left[n^{2}(Q / P)-1\right][(T / P)-1] \\
-x_{0}^{2}\left[n^{2}(N / P)-1\right]-y_{0}^{2}\left[n^{2}(Q / P)-1\right]=0 .
\end{gathered}
$$

It is easy to prove that the original straight-line form of the bar corresponds to stable equilibrium if the compressive force $P$ is sufficiently small, since by decreasing 
$P$ the first three terms on the right hand side of Eq. (12) can be made positive and large as compared to the last two terms. In the investigation of the stability of the bar under increasing values of $P$ it is to be noted that if any of the inequalities (14)(16) is satisfied for $n=k$, it is also satisfied for $n=k+p$, where $k$ and $p$ are arbitrary positive integers. Consequently the bar is stable if, and only if, inequalities (14)-(16) are satisfied when $n=1$. The smallest critical load can be calculated from Eqs. (17)(19) if 1 is substituted for $n$. This load alone is of practical importance unless geometric constraints (for instance rigid end fixation or additional supports between the ends of the bar) prevent displacement corresponding to the first terms of the Fourier series in Eqs. (1a)-(1c). In such a case the smallest value of $n$ that is compatible with the restraints must be used in Eqs. (17)-(19) for the calculation of the buckling load, for instance $n=2$ when the ends of the bar are prevented from rotating and warping.

Eqs. (17)-(19) permit a discussion of the various types of buckling of bars of different cross section. With an asymmetric section $x_{0} \neq 0$ and $y_{0} \neq 0$. In this case with increasing $P$ a value is reached at which Eq. (19) is fulfilled while the left sides of Eqs. (17) and (18) are still greater than zero. The buckling load $P$ can be calculated from Eq. (19) which is a cubic in $(1 / P)$. The deflection pattern is flexural-torsional since it contains the non-vanishing coefficients $a_{n}, b_{n}$, and $c_{n}$ simultaneously.

If the section has one plane of symmetry, one of the coordinates of the centroid, say $x_{0}$, vanishes. Then Eq. (19) reduces to

$$
\left[n^{2}(Q / P)-1\right]\left\{\rho^{2}\left[n^{2}(N / P)-1\right][(T / P)-1]-y_{0}^{2}\right\}=0 .
$$

Consequently two distinct types of buckling are possible. One is purely flexural and symmetric. It corresponds to a buckling load which is the solution of Eq. (18). The other is flexural-torsional since it simultaneously contains displacement components corresponding to the non-vanishing coefficients $a_{n}$ and $c_{n}$; it is antisymmetric; its buckling load $P$ is the (smaller) root of the quadratic in $(1 / P)$ that can be obtained by dividing Eq. (20) by $\left[n^{2}(Q / P)-1\right]$. The smaller of the two distinct buckling loads is of practical importance. The buckling load according to Eq. (17) is always greater than the smaller root of the quadratic.

Finally, if the section is doubly symmetric or point symmetric, $x_{0}=y_{0}=0$. Then Eq. (19) reduces to

$$
(T / P)-1=0 .
$$

Buckling is either purely flexural or purely torsional. Of practical importance is the smallest of the solutions for $P$ of the three Eqs. (17), (18), and (21). 